\title{
Dependence on Ethylene of the Induction of Phenylalanine Ammonia- Lyase Activity in Rice Leaf Infected with Blast Fungus ${ }^{\dagger}$
}

\author{
Minoru Haga, Tetsuya Haruyama, Hiromasa Kano, \\ Yasuharu SeKIZAWA, ${ }^{\dagger \dagger}$ Sueo URUSHIzAKI* \\ and Kuniomi Matsumoto** \\ Laboratory of Applied Biochemistry, Department of Agricultural Chemistry, \\ Faculty of Agriculture, Tamagawa University, \\ Machida-shi, Tokyo 194, Japan \\ * Laboratory of Plant Growth Regulators, National Institute of Agrobiological Resources, \\ The Ministry of Agriculture, Forestry and Fisheries, \\ Yatabe-cho, Tsukuba-gun, Ibaraki 305, Japan \\ ** Laboratory of Agricultural and Industrial Chemicals, The Research Laboratories, \\ Meiji Seika Kaisha Ltd., Morooka-cho, Kohoku-ku, \\ Yokohama 222, Japan
}

Received October 5, 1987

\begin{abstract}
The application of ethephon (ETP), an ethylene generating chemical, to press-injured rice leaves induced PAL (EC 4.3.1.6) activity. This induction disappeared when the concentration was low. A Scatchard plot for ligand binding gave a linear relationship between the PAL reaction rate at the time for the maximum rate of induction $\left(t_{\mathrm{m}}\right)$ along the sigmoidal progress and the ratio of the PAL reaction rate versus ETP in a specific range of concentration. A similar application of glutamate (Glu) induced PAL activity, and the application of 1-aminocyclopropane-1-carboxylic acid (ACC) also induced PAL activity. The induction of rice leaf PAL with Glu was inhibited in the presence of tiron (TR), a superoxide scavenger, was weakly inhibited with aminooxyacetic acid (AOA), but was not inhibited with abscisic acid (ABA). The induction with ACC was also inhibited in the presence of TR, was weakly inhibited with AOA, and further was weakly inhibited with ABA. The inoculation of blast fungus conidia or apphcation of fungal proteoglucomannan (RIF) to press-injured rice leaves induced PAL activity. The induction proceeded along sigmoidal curve accompanying the distinct auto-inactivation phase. Each approximated $t_{\mathrm{m}}$ (midpoint of an individual sigmoidal curve) was determined; $t_{\mathrm{m}}=2.4 \mathrm{hr}$ with an incompatible race, $t_{\mathrm{m}}=5.3 \mathrm{hr}$ with a compatible race, and $t_{\mathrm{m}}=11.0 \mathrm{hr}$ with RIF stimulation. The 3 inhibition profiles of TR, AOA and ABA upon rice leaf PAL induction either with inoculation or with stimulation were quite similar to the inhibition profiles upon PAL induction with Glu.
\end{abstract}

The inductions of PAL and peroxidase in rice leaves infected with blast fungus have been reported by Matsuyama and Kozaka. ${ }^{1)} \mathrm{A}$ high-

er rate of ethylene evolution at an early stage of rice blast infection on a resistant cultivar has been also observed by Kozaka and

+ Causality Analysis on Cascades of Reactions in the Induced Defense Mechanism of Rice Plant. Part IV. This work was supported in part by a Grant-in-Aid for Research from the Agriculture, Forestry and Fisheries Research Council, Japan.

${ }^{\dagger}$ To whom inquiries and requests for reprints should be addressed.

Abbreviations: PAL, phenylalanine ammonia-lyase; Cin, cinnamate; RIF, resistant-reaction inducing factor (stimulant, blast fungus hyphal component or proteoglucomannan in previous papers); TR, tiron (1,2-dihydroxybenzene-3,5-disulfonic acid disodium salt monohydrate); AOA, amino-oxyacetic acid (carboxy-methoxylamine hemihydrochloride); CY, cycloheximide; ABA, abscisic acid; ACC, 1-aminocyclopropane-1-carboxylic acid; Glu, glutamate monosodium salt; $\alpha$-KGA, $\alpha$-ketoglutarate disodium salt; ETP, ethephon (2-chloroethyl phosphonic acid); IAA, $\beta$-indoleacetic acid. 
Teraoka. ${ }^{2)}$ The dependence of peroxidase induction on ethylene in sweet potato tuberous root has been demonstrated by Imaseki. ${ }^{3}$ Stahmann et al. have proposed an attractive hypothesis that endogenous ethylene induces resistant characteristics in sweet potato tuberous root concomitantly with the peroxidase induction. ${ }^{4)}$ In this paper, to elucidate the dependence of PAL induction on ethylene in rice leaves infected with blast fungus, time course surveys of the induction of rice leaf PAL with press-injured application of either ETP, an ethylene generating chemical, Glu and $\mathrm{ACC}$ were made. The inhibition spectra of the induction of rice leaf PAL either with Glu or ACC were determined using TR (a superoxide scavenger ${ }^{5,6}$ ), AOA to (an inhibitor to the pyridoxal enzyme ${ }^{7)}$, and ABA (an inhibitor to the induction of ACC synthase ${ }^{8)}$ ). Since the dynamic behavior of superoxide generation at a very early stage by rice leaves infected with blast fungus has already been elucidated, ${ }^{9)}$ and the conversion of ACC to ethylene in the methionine pathway, which is ubiquitous in higher plants, has been reported to be dependent on endogenous superoxide, ${ }^{5,6)}$ TR was used in this study to determine the dependence of ethylene generation on the superoxide anion. Time course surveys were also made of the induction of rice leaf PAL with press-injured inoculation of either an incompatible or compatible blast fungus conidia, and with press-injured stimulation of RIF. After the determination of $t_{\mathrm{m}}$ (the time for the maximum rate of induction after inoculation or stimulation, corresponding to the midpoint of an individual sigmoidal curve), the inhibition spectra of the induction of rice leaf PAL with inoculation of either the incompatible or compatible conidia, or with stimulation by RIF, were determined using TR, AOA, ABA or CY, and were compared with the inhibition spectra obtained with Glu or $\mathrm{ACC}$, which are precursors for ethylene biosynthesis. All experimental data were collectively evaluated to discuss the dependence upon ethylene of PAL induction in rice leaves infected with blast fungus.

\section{MATERIALS AND METHODS}

Chemicals. TR was obtained from Fisher Scientific Co., Fair Lawn, NJ. AOA, ABA, CY, ACC and $\alpha$-KGA were obtained from Sigma Chemical Co., St. Louis, MO. Phenylalanine and Glu were obtained from Wako Pure Chemical Industries Ltd., Tokyo, and ETP was a product of Nissan Chemical Industries Ltd., Tokyo.

Blast fungus races and preparation of conidia. Blast fungus (Pyricularia oryze CAVARA) H-373 (race No. 007) and $\mathrm{TH}$ 68-85 (race No. 031) were used as previously described. ${ }^{9)}$ The conidia were also prepared as previously described. ${ }^{9 \text { \} }}$

Rice seedlings and their inoculation with blast fungus conidia or stimulation with RIF. The rice plant used (cultivar: Jukkoku), its genetic characteristics, the culture conditions and the inoculation with blast fungus conidia or stimulation with RIF were the same as previously described. ${ }^{9)}$ The rice leaves used for preparing the PAL source were the 4 th leaves, which were completely developed.

Preparation of PAL source. One hundred mg of pressinjured 4th leaves that had been treated with chemicals and/or inoculated were cut into pieces and homogenized in a Biotron (BT 10/20 3500, Switzerland) in $3 \mathrm{ml}$ of $0.1 \mathrm{M}$ potassium dihydrogen phosphate buffer at $\mathrm{pH} 6.5$ and below $5{ }^{\circ} \mathrm{C}$ containing $20 \mathrm{~mm}$ dithiothreitol, $2 \%$ (v/v) glycerol and $0.01 \%$ Triton $\mathrm{X}-100$. The homogenate was centrifuged at $23,000 \times g$ and $0^{\circ} \mathrm{C}$ for $20 \mathrm{~min}$. The supernatant obtained was used for the crude enzyme source of PAL. ${ }^{10)}$

Determination of $P A L$ activity. The reaction mixture contained $20 \mu \mathrm{mol}$ phenylalanine in $100 \mu \mathrm{mol}$ sodium borate buffer at $\mathrm{pH} 8.8$ to a total volume of $3.9 \mathrm{ml}^{10}{ }^{10}$ The reaction was started at $40^{\circ} \mathrm{C}$ by adding $0.1 \mathrm{ml}$ of the crude enzyme source already described. The reaction was continued for 5 to $10 \mathrm{~min}$ within a $5 \%$ decrease of phenylalanine. The reaction was stopped by the addition of 0.5 $\mathrm{ml}$ of $5 \mathrm{~N} \mathrm{HCl}$. The absorbance at $225 \mathrm{~nm}$ and $270 \mathrm{~nm}$ was determined with a Shimadzu UV-260 recording spectrophotometer in conjunction with a Shimadzu HCC-260 temperature-controlled cell holder and MC303 multistirer. For the blank run, a reaction mixture without phenylalanine was used at the reference position after adding $0.5 \mathrm{ml}$ of $5 \mathrm{~N} \mathrm{HCl}$. Using the absorption coefficients of phenylalanine $\left(0.0824 \mathrm{~mm}^{-1} \mathrm{~cm}^{-1}\right.$ at $255 \mathrm{~nm}$ and $0.0129 \mathrm{~mm}^{-1} \mathrm{~cm}^{-1}$ at $\left.270 \mathrm{~nm}\right)$ and of transcinnamic acid $\left(0.0057 \mathrm{~mm}^{-1} \mathrm{~cm}^{-1}\right.$ at $255 \mathrm{~nm}$ and 0.0079 $\mathrm{mM}^{-1} \mathrm{~cm}^{-1}$ at $270 \mathrm{~nm}$ ), the concentration of phenylalanine or trans-cinnamic acid was calculated by the usual simultaneous equations. 
Approximation of $t_{\mathrm{m}}$ (time to the maximum rate of rice leaf PAL induction after inoculation or stimulation). As the induction of PAL progressed along a sigmoidal curve for the time axis, the time for the maximum rate of induction coincided with the midpoint of the sigmoidal curve. The midpoint of a given sigmoidal curve was mathematically approximated as previousiy described. ${ }^{11)}$

\section{RESULTS}

Induction of rice leaf PAL with ETP and the Scatchard plot for ligand binding

On a 4th rice leaf that had just developed, press-injured spots ( $2 \mathrm{~mm}$ in diameter) were made in 2 progressions along both sides of the leaf central rib. One progression had 12 pressinjured spots at intervals of $1 \mathrm{~cm}$. Five $\mu \mathrm{l}$ of ETP aqueous solution was applied to each press-injured spot, and the time-course determination of the induction of rice leaf PAL was started.

The results are summarized in Fig. 1. The approximated $t_{\mathrm{m}}$ values were $11.6 \mathrm{hr}$ for 50 ppm, $6.6 \mathrm{hr}$ for $100 \mathrm{ppm}, 7.5 \mathrm{hr}$ for $250 \mathrm{ppm}$ and $8.3 \mathrm{hr}$ for $500 \mathrm{ppm}$. These results indicate that exogenous ethylene induced rice leaf PAL. From classic Michaelis-Menten types of

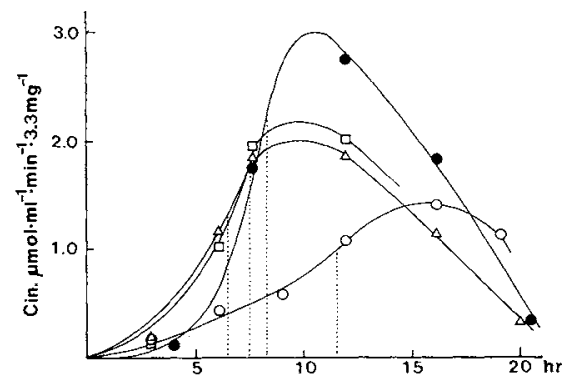

FIG. 1. Dynamic Behavior of Rice Leaf PAL Induction with ETP.

Ordinate: rate of PAL activity.

Abscissa: time after the press-injured application of ETP. O-O, application of $50 \mathrm{ppm}(0.35 \mathrm{mM})$ ETP, $t_{\mathrm{m}}$ approximated to $11.6 \mathrm{hr}$ as indicated with the dotted vertical line; $\triangle-\triangle$, application of $100 \mathrm{ppm}(0.69 \mathrm{~mm})$ ETP, $t_{\mathrm{m}}$ approximated to $6: 6 \mathrm{hr} ; \square-\square$, application of $250 \mathrm{ppm}$ $(1.74 \mathrm{~mm}) \mathrm{TEP}, t_{\mathrm{m}}$ approximated to $6.9 \mathrm{hr} ;-0$, application of $500 \mathrm{ppm}(3.46 \mathrm{~mm}) \mathrm{ETP}, t_{\mathrm{m}}$ approximated to $8.3 \mathrm{hr}$.

PAL activity in the merely press-injured run was subtracted from the corresponding runs as a blank. plots of the application of a lower concentration of ETP than $50 \mathrm{ppm}(0.35 \mathrm{~mm})$, the repeated preliminary experiments indicated that the application of a concentration lower than $16.2 \mathrm{ppm}(0.11 \mathrm{~mm})$ did not induce PAL. After subtracting $0.11 \mathrm{~mm}$ from exogenously applied concentrations of ETP, an effective dose, $[\mathrm{He}]_{\mathrm{ef}}$, was obtained, and the relationship between the initial rate of PAL activity at $t_{\mathrm{m}}, V_{\mathrm{PAL}}$ on the abscissa, and the ratio of $V_{\mathrm{PAL}} /[\mathrm{He}]_{\mathrm{ef}}$ on the ordinate were plotted to obtain a Scatchard plot $^{12)}$ for ligand binding. The result is shown in Fig. 2. The Scatchard plot for ligand binding gave a linear relationship in a specific range of ETP concentration. The result indicates that the induction of rice leaf PAL activity by the application of ETP was a phenomenon of "saturation" in a specific range of ETP concentration, like the formation of an enzyme-substrate complex. The apparent dissociation constant $\left(K_{\mathrm{s}}\right)$ calculated was $2.35 \times 10^{-7} \mathrm{M}$, when the putative binding sites had been tentatively set at 1 binding site per functional proteinic unit.

Induction of rice leaf $P A L$ with Glu or $A C C$ and the inhibition experiment with $T R, A O A$ and $A B A$

With similar press-injured application to



Fig. 2. Scatchard Plot for Ligand Binding between $[\mathrm{He}]_{\mathrm{ef}}$ and $V_{\mathrm{PAL}}^{\mathrm{rat}}$ at $t_{\mathrm{m}}$.

Ordinate: $V_{\mathrm{PAL}} /[\mathrm{He}]_{\mathrm{ef}}$, where $V_{\mathrm{PAL}}$ was the initial rate of PAL activity at $t_{\mathrm{m}}$ and $[\mathrm{He}]_{\mathrm{ef}}$ was the effective dose of ETP. Abscissa: $V_{\mathrm{PAL}}$

$[\mathrm{He}]_{\mathrm{ef}}$ of ETP was $0.06,0.13,0.17,0.24,0.33,0.58,1.62$, 2.25 or $3.35 \mathrm{mM}$ from the left-hand side. 
that for ETP already described, it was observed that the application of a $5 \mu$ l solution containing $1 \mathrm{mM}$ Glu induced rice leaf PAL activity, and that the application of a $5 \mu 1$ solution containing $1 \mathrm{~mm}$ ACC also induced rice leaf PAL activity. In the rice leaf system

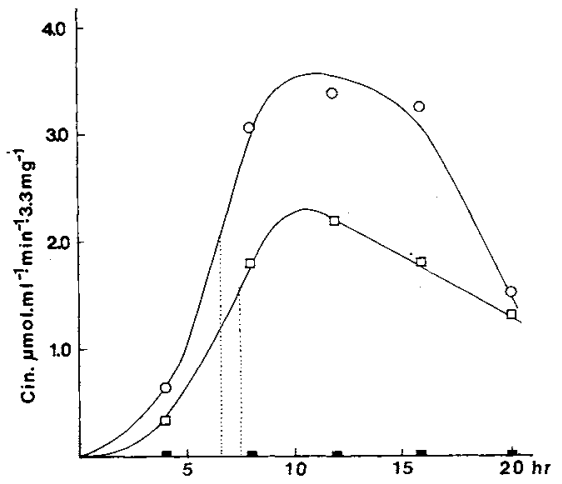

FIG. 3. Dynamic Behavior of Rice Leaf PAL Induction with Glu or ACC.

Ordinate: rate of PAL activity.

Abscissa: time after the application of a $5 \mu$ l solution of $1 \mathrm{~mm}$ Glu or ACC on a press-injured spot $(2 \mathrm{~mm}$ in diameter).

The application procedure was similar to that for ETP, see the text. The dotted vertical lines show the individual midpoint approximated for the sigmoidal progress of PAL induction.

$\square-\square$, Glu; O-O, ACC; $\square-\square \alpha-\mathrm{KGA}$.

$\mathrm{PAL}$ activity in the merely press-injured run was subtracted from the corresponding runs as a blank.

Table I. InHibitory EfFEct OF TR, AOA AND ABA ON THE INDUCTION OF RICE LEAF PAL With Press-injured Application OF Glu OR ACC

\begin{tabular}{cccc}
\hline $\begin{array}{c}\text { Substrate } \\
\text { for } \\
\text { PAL induction }\end{array}$ & \multicolumn{3}{c}{ Inhibition $\%$ at $t_{\mathrm{m}}$} \\
\cline { 2 - 4 } & $\mathrm{TR}$ & $\mathrm{AOA}$ & $\mathrm{ABA}$ \\
\hline Glu & 83.0 & 33.6 & 0 \\
$\mathrm{ACC}$ & 97.0 & 32.5 & 36.0 \\
\hline
\end{tabular}

Application procedure for the inhibitors: (1) $5 \mu \mathrm{l}$ of $500 \mathrm{ppm}$ TR, $500 \mathrm{ppm}$ AOA or $50 \mathrm{ppm} \mathrm{ABA}$ was respectively mounted on the press-injured spots $(2 \mathrm{~mm}$ in diameter), (2) and after $4 \mathrm{hr}$ standing, $5 \mu \mathrm{l}$ of $1 \mathrm{~mm}$ Glu or ACC containing TR (100 ppm), AOA (100 ppm) or ABA $(50 \mathrm{ppm})$ was mounted, and (3) the homogenization medium also contained TR (100 ppm), AOA (100 ppm) or ABA (50 ppm). used for this study, the application of a similar concentration of $\alpha-\mathrm{KGA}$ did not induce rice leaf $\mathrm{PAL}$, these results being shown in Fig. 3. The approximated $t_{\mathrm{m}}$ for the induction of PAL with Glu was $7.5 \mathrm{hr}$, and that for the induction of PAL with $\mathrm{ACC}$ was $6.6 \mathrm{hr}$.

When $5 \mu \mathrm{l}$ of a solution of TR $(500 \mathrm{ppm})$, AOA $(500 \mathrm{ppm})$ or ABA $(50 \mathrm{ppm})$ was respectively mounted on the press-injured spots ( $2 \mathrm{~mm}$ in diameter), and after $4 \mathrm{hr}$ of standing, $5 \mu \mathrm{l}$ of a $1 \mathrm{~mm}$ Glu or ACC solution containing TR $(100 \mathrm{ppm})$, AOA $(100 \mathrm{ppm})$ or ABA $(50 \mathrm{ppm})$ was applied with a microsyringe. The homogenization medium also contained TR (100 ppm), AOA (100 ppm) or ABA (50 ppm) to prevent a rebound effect, and the inhibition percentage given by each inhibitor is summarized in Table I. It is to be noted that (1) TR, a superoxide scavenger, markedly inhibited the induction of rice leaf PAL either with Glu or ACC, (2) ABA did not inhibit PAL induction with Glu, but weakly inhibited this with ACC, and (3) AOA weakly inhibited this either with Glu or ACC.

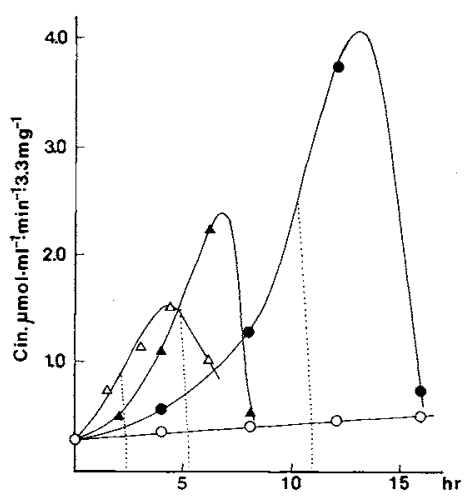

FIG. 4. Dynamic Behavior of Rice Leaf PAL Induced by Inoculating with an Incompatible or Compatible Blast Fungus Conidia or with the Stimulation by RIF.

Ordinate: rate of PAL activity.

Abscissa: time after press-injured inoculation or stimulation. Number of conidia in the inoculum: $2.0 \times 10^{5}$ conidia/ml water. Concentration of RIF applied: $10 \mathrm{mg}$ of partially purified $\mathrm{RIF} / \mathrm{ml}$ water.

$\triangle-\triangle$, incompatible inoculation; $\mathbf{\Delta}-\mathbf{\Delta}$, compatible inoculation; - - RIF stimulation; $\mathrm{O}_{-}^{-} \mathrm{O}$, merely pressinjured. 

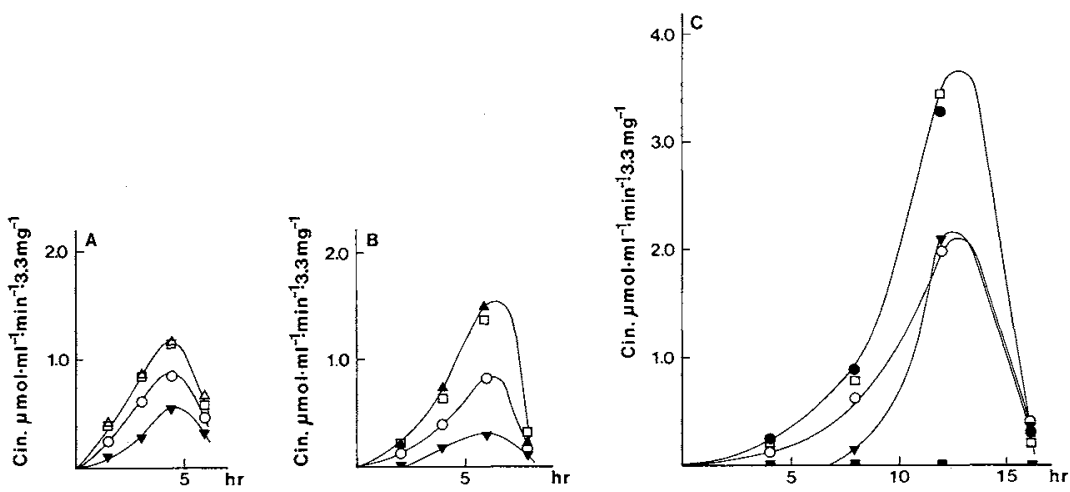

FIG. 5. Inhibitory Effect of TR, AOA, ABA and CY on the Induction of Rice Leaf PAL with Press-injured Inoculation of an Incompatible or Compatible Blast Fungus Conidia or with Press-injured RIF Stimulation.

Ordinate: rate of PAL activity.

Abscissa: time after press-injured inoculation or stimulation.

A: $\triangle-\triangle$, incompatible inoculation; B: $\mathbf{\Delta}-\boldsymbol{\Delta}$, compatible inoculation; $\mathrm{C}$ :

TR inhibition; $\mathrm{O}-\mathrm{O}$, AOA inhibition;

, ABA inhibition;

, CY.

PAL activity in merely the press-injured run with or without an individual inhibitor was subtracted from the corresponding runs as a blank.

TABLE II. INHIBITORY EFFECT OF TR, AOA, ABA OR CY ON THE INDUCTION OF RICE LEAF PAL WITH Press-INJURED INOCULATION OF AN INCOMPATIBLE or Compatible Blast Fungus Conidia, or With PRess-INJURed RIF Stimulation

\begin{tabular}{lcccc}
\hline $\begin{array}{c}\text { Inoculation } \\
\text { or } \\
\text { stimulation }\end{array}$ & TR & AOA & ABA & $\mathrm{CY}$ \\
\cline { 2 - 5 } & & \multicolumn{4}{c}{ Inhibition $\%$ at $t_{\mathrm{m}}$} \\
\hline Incompatible & 69.8 & 35.7 & 0 & $\mathrm{NT}^{*}$ \\
Compatible & 60.9 & 42.3 & 0 & $\mathrm{NT}$ \\
RIF & 52.5 & 38.9 & 0 & 100 \\
\hline
\end{tabular}

* NT, not tested; the number of conidia in the inoculum and the concentration of RIF applied were same as those Fig. 4.

The application procedure for the inhibitors was the same as that in Table I; CY (20 ppm) was applied, after $4 \mathrm{hr}$ standing, CY (10 ppm) was mixed with RIF solution and applied. The homogenization medium also contained CY (10 ppm).

Induction of rice leaf PAL by inoculating with an incompatible or compatible blast fungus conidia, by stimulating with RIF, and by the inhibition experiments with $T R, A O A, A B A$ and $C Y$.

With similar press-injured application to that for ETP already described, it was observed that the inoculation with a $5 \mu$ l aqueous suspension of an incompatible or compatible
TABLE III. INHIBITORY EFFECT OF TR, AOA AND ABA ON Rice LEAF PAL INDUCED WITH Press-INJURED RIF Stimulation

\begin{tabular}{ccc}
\hline Inhibitor & $\begin{array}{c}\text { Concentration } \\
\text { (ppm in reaction } \\
\text { mixture) }\end{array}$ & $\begin{array}{c}\text { Inhibition \% } \\
\text { (with initial } \\
\text { velocity) }\end{array}$ \\
\hline TR & 100 & 6.9 \\
& 200 & -3.5 \\
AOA & 100 & -6.7 \\
& 200 & 4.6 \\
ABA & 10 & -3.9 \\
& 20 & 6.2 \\
CY & 5 & -3.9 \\
& 10 & 6.2 \\
\hline
\end{tabular}

PAL induction in the merely press-injured run with or without an individual inhibitor was subtracted from the corresponded runs.

blast fungus conidia, or stimulation with RIF induced the rice leaf PAL. These results are shown in Fig. 4. The approximated $t_{\mathrm{m}}$ for the induction of PAL with incompatible inoculation was $2.4 \mathrm{hr}$, that with compatible inoculation was $5.3 \mathrm{hr}$, and that with RIF stimulation was $11.0 \mathrm{hr}$. This orderliness in the incompatible and compatible inoculations and the RIF stimulation coincided with rice leaf 
peroxidase induction, ${ }^{13)}$ but the induction of rice leaf PAL was generally determined to be an earlier phenomenon when compared with the induction of peroxidase. The orderliness within the incompatible and compatible inoculation also coincided with superoxide generation (incompatible $t_{\mathrm{m}}, 0.5 \mathrm{hr}$; compatible $\left.t_{\mathrm{m}}, 2.9 \mathrm{hr}\right){ }^{9\}}$

The inhibitory effects of TR, AOA, ABA and $\mathrm{CY}$ on the induction of rice leaf PAL with press-injured ihoculation of incompatible or compatible blast fungus conidia, and with press-injured RIF stimulation were determined with a similar procedure to that already described, the results being summarized in Table II and Fig. 5. It is to be noted that the inhibition spectra comprising TR, AOA and ABA were quite similar to that for rice leaf PAL induction with Glu that has already been described, and that PAL induction with RIF stimulation was completely inhibited with $\mathrm{CY}$ when $20 \mathrm{ppm}$ of CY was applied $4 \mathrm{hr}$ before stimulation, and when $10 \mathrm{ppm}$ was applied with RIF stimulation. The latter indicates that PAL induction was operated and regulated by de novo synthesis. The pretreatment of rice seedlings with TR, AOA or ABA did not interfere with the infection of blast fungus.

Direct inhibitory effects of $T R, A O A$ and $A B A$ on rice leaf $P A L$ activity

The direct inhibitory effects of TR, AOA, $\mathrm{ABA}$ and $\mathrm{CY}$ on the activity of rice leaf PAL was determined near the maximum rate of PAL activity, when rice leaf PAL was induced with press-injured RIF stimulation. The results are summarized in Table III, and indicate that the enzymic activity of PAL itself was inhibited neither by TR, AOA, ABA nor $C Y$. This revealed that $T R, A O A, A B A$ and $C Y$ inhibited the process of rice leaf PAL induction. The concentration of individual inhibitor in the reaction mixture, which was introduced with the PAL source (Tables I and II), was estimated as being a few ppm, because of extensive dilution of the PAL source.

\section{DISCUSSION}

Since Stahmann et $a l^{4)}$ proposed an attractive hypothesis for the induction of resistant reactions in sweet potato tuberous root against black rot disease (causal organism, Ceratocystis fimbriata), in which ethylene may have been the trigger to result in the induction of resistant reactions that inhibited further spread of the pathogen, there have been many reports describing that ethylene might participate in the induction of resistant reactions. ${ }^{14 \sim 17)}$ On the contrary, there are opposing opinions that ethylene may merely relate to the formation of disease symptoms, and that ethylene may rather relate to the establishment of infections. ${ }^{18 \sim 22)}$ Researches in this respect on the rice plant have been originated by an unexpected finding that the efficacy of probenazol, a non-fungicidal protectant, against rice blast is clearly counteracted by pretreating with ABA. $^{23)}$ At that time, Yoshii and Imasek $\left.i^{8}\right)$ have already demonstrated that ABA clearly counteracted the induction of ACC synthase (pyridoxal enzyme) activity with IAA. Matsumoto et al. $^{24)}$ and Iwata et $a l^{25)}$ have further demonstrated that the suppression of rice blast incidence by IAA or ethephon was strongly counteracted by the application of ABA prior to inoculation, and that the negative correlation coefficient between peroxidase induction and the decrease of blast incidence was $-82 \%$.

In the present report, it was first experimentally demonstrated that the induction of rice leaf PAL activity clearly depended on exogenous ETP, an ethylene generating chemical, indicating linear relationships in the Scatchard plot for ligand binding in a specific range of exogenous ETP concentration (Figs. 1 and 2). It was also demonstrated that rice leaf PAL induction was accomplished with exogenous Glu or ACC but not with $\alpha$-KGA (Fig. 3). Originally, the glutamate pathway for ethylene biosynthesis was operable within such microorganisms as Penicillium digitatum, ${ }^{26)}$ Pseudomonas syringae $\mathrm{pv.}$ phaseolicola $^{27)}$ and Pseudomonas syringae pv. 
glycinea ${ }^{28)}$ but recently, Hyodo and Uritani $^{29)}$ have suggested that the pathway for ethylene biosynthesis in infected sweet potato root tissue may differ from the methionine pathway in which ACC serves as an intermediate in the adjacent healthy tissue; they emphasized that the possiblity of a glutamate pathway in the infected tissue should be investigated by future study. In the present study, the inhibition spectrum obtained by the application of TR, AOA and ABA on the induction of rice leaf PAL with Glu was different from that obtained with $\mathrm{ACC}$, and the induction of PAL with Glu was not inhibited by the application of ABA (Table I). It seems likely that about $36 \%$ of the PAL induction with $\mathrm{ACC}$ might depend on the inductive formation of ACC synthase (Table I and Fig. 3). The inhibition spectra obtained by the application of TR, AOA and ABA on the induction of rice lice leaf PAL activity with incompatible or compatible inoculation or with RIF stimulation were quite similar to that with Glu (Figs. 4, 5 and Table II). The determination of $t_{\mathrm{m}}$ for rice leaf PAL induction with an incompatible or compatible fungus race, or with RIF stimulation, indicated that PAL induction was quite an early phenomenon when compared with those $t_{\mathrm{m}}$ values for rice leaf peroxidase induction. ${ }^{13)}$ These observations bear out the suggestion that the glutamate pathway may operate for the initiation reaction of endogenous ethylene generation $^{29 \sim 31)}$ in infected rice leaf tissue. On the other hand, the marked inhibition of rice leaf PAL induction with TR indicated the inyolvement of a superoxide anion and/or the derived active oxgen species in the ethylene biosynthesis both with the glutamate and methionine pathway ${ }^{5,6,9,13,32)}$ (Tables I, II, III and Fig. 5). The explanation for weak inhibition given by the application of AOA on PAL induction with Glu is uncertain at present, but it might be related to aminotransferases (pyridoxal enzymes) partially concerned with the conversion of $\alpha-\mathrm{KGA}$ to Glu. It is generally accepted that (1) both PAL and peroxidase play indispensable roles in operat- ing the hydroxycinnamate pathway leading to lignin for the formation of a physical barrier against phytopathogenes, and (2) PAL functions to feed cinnamate to the pathway and peroxidase functions to polymerize oxidatively the resultant hydroxycinnamates. ${ }^{30)}$ The ABA sensitive characteristics for the induction of rice leaf peroxidase will be reported relevant to the operation of a hydroxycinnamate pathway in the forthcoming paper.

Acknowledgments. The authors are grateful to Dr. Kunio Toriyama, former Director, National Institute of Agrobiological Resources, Tsukuba, for his invaluable suggestion relating to the resistance characteristics of rice plant, and also to Professor Dr. Yoshinori Takino, Graduate School, Faculty of Agriculture, Tamagawa University.

\section{REFERENCES}

1) N. Matsuyama and T. Kozaka, Ann. Phytopathol. Soc. Jpn., 36, 368 (1970).

2) T. Kozaka and T. Teraoka, Ann. Phytopathol. Soc. Jpn., 43, 549 (1977).

3) H. Imaseki, Plant Physiol., 46, 172 (1970).

4) M. A. Stahmann, B. G. Clare and W. Woodbury, Plant Physiol., 41, 1505 (1966).

5) R. L. Legge, J. E. Thompson and J. E. Baker, Plant and Cell Physiol., 23, 171 (1982).

6) D. G. MacRae, J. E. Baker and J. E. Thompson, Plant and Cell Physiol., 23, 375 (1982).

7) D. O. Adams and S. F. Young, Trends in Biochem. Sci., 6, 161 (1981).

8) H. Yoshii and H. Imaseki, Plant and Cell Physiol., 22, 369 (1981).

9) Y. Sekizawa, M. Haga, E. Hirabayashi, N. Takeuchi and Y. Takino, Agric. Biol. Chem., 51, 763 (1987).

10) J. Koukol and E. E. Conn, J. Biol. Chem., 236, 2692 (1961).

11) Y. Sekizawa, I. Komiya, M. Iwata, T. Watanabe, S. Murata and K. Umemura, J. Pesticide Sci., 8, 121 (1983).

12) I. H. Segel, "Biochemical Calculations" (Jpn. version), John Wiley \& Sons Inc., New York/Hirokawa Shoten, Tokyo, 1982, pp. $272 \sim 274$.

13) Y. Sekizawa, M. Haga, M. Iwata, A. Hamamoto, C. Chihara and Y. Takino, J. Pesticide Sci, 10, 225 (1985)

14) L. C. van Loon, "Active Defense Mechanism in Plants," Plenum Press, New York, 1982, pp. $247 \sim 273$.

15) A. Toppan, A. D. Roby and M. Esquerre-Tugaye, Plant Physiol., 70, 82 (1982).

16) A. M. M. de Laat and L. C. van Loon, Physiol. Plant 
Pathol., 22, 261 (1983).

17) O. H. Jones, Phytochemistry, 23, 1349 (1984).

18) J. M. Daly, P. M. Seuvers and P. Ludden, Phytopathology, 60, 1648 (1970).

19) L. Sequeira, "Hormone Metabolism in Diseased Plants," Vol. 24, Ann. Rev. Plant Physiol., 1973, pp. $353 \sim 379$.

20) A. Gentile and A. Matta, Physiol. Plant Pathol., 5, 27 (1975).

21) D. K. Cronshow and G. F. Pegg, Physiol. Plant Pathol., 9, 33 (1976).

22) H. Yamamoto and T. Tani, Ann. Phytopathol. Soc. Jpn., 52, 460 (1986).

23) Y. Sekizawa, "Mode of Action of Rice Blast Protectant, Probenazole," in IUPAC Pesticide Chemistry III, ed. by J. Miyamoto et al., Pergamon Press, Oxford, 1983, pp. 147 152.

24) K. Matsumoto, Y. Suzuki, S. Mase, T. Watanabe and Y. Sekizawa, Ann. Phytopathol. Sóc. Jpn., 46, 307 (1980).

25) M. Iwata, Y. Sekizawa, H. Iwamatsu, Y. Suzuki and T. Watanabe, Ann. Phytopathol. Soc. Jpn., 47, 646
(1981).

26) T. W. Chou and S. F. Yang, Arch. Biochem. Biophys., 157, 73 (1973).

27) M. Goto and H. Hyodo, Plant and Cell Physiol., 28, 405 (1987).

28) M. Sato, S. Urushizaki, K. Nishiyama, F. Sakai and Y. Ota, Agric. Biol. Chem., 51, 1177 (1987).

29) H. Hyodo and I. Uritani, Plant and cell Physiol., 25, 1147 (1984).

30) Y. Sekizawa, "Defense Mechanism of Rice Plant: A Case of Rice Blast-Working Hypothesis," Abstracts of Papers, The Annual Meeting of The Japanese Soc. of Plant Physiologist (Sendai); symposium 1S-3, 1986, p. 26.

31) S. F: Yang, "Mechanism and Regulation of Ethylene Biosynthesis" in The new Frontiers in Plant Biochemistry, ed. by T. Akazawa and H. Imaseki, Jpn. Scientific Soc. Press, Tokyo, 1.983, pp. $133 \sim 151$.

32) M. Haga, Y. Sekizawa, M. Ichikawa, H. Hiramatsu, A. Hamamoto, Y. Takino and M. Ameyama, Agric. Biol. Chem., 50, 1427 (1986). 